\section{The Rodger Winn Award}

JNCCN Staff

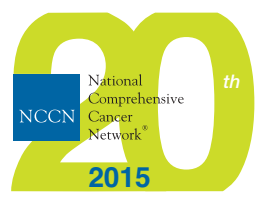

Over the past 20 years, staff at NCCN have had the pleasure of working with many thoughtful and dedicated oncologists and other health care professionals, as well as patient advocates and patients themselves. Perhaps one of the most memorable was Rodger Winn, MD. As was noted in last month's NCCN Anniversary Reflections, Dr. Winn was both the "father" of the NCCN Clinical Practice Guidelines in Oncology (NCCN Guidelines) and the founding Editor-in-Chief of JNCCN. He was an enthusiastic advocate for patients, fellow health care providers, and NCCN itself, and he continued working to further the mission of improving the lives of patients with cancer, even through his own cancer diagnosis and treatment, until his death in 2007.

To celebrate his life and dedication, each year NCCN gives The Rodger Winn Award to one NCCN Guidelines Panel Member who exemplifies Dr. Winn's leadership, drive, and commitment to the development of evidence-based guidelines tempered by expert judgment. The recipient provides a voice for the core mission of NCCN, improving the quality of care for patients. Specifically, the award recognizes service in the development of clinical practice guidelines, promotion of collegiality in NCCN activities, commitment to excellence, and dedication to multidisciplinary care.

To help mark the 20th Anniversary of the organization that Dr. Winn helped begin, JNCCN asked some of the past recipients of the Rodger Winn Award to talk about the role of NCCN:

\section{David S. Ettinger, MD (2009)}

"It has been an honor for me to be associated with NCCN all these years, to see how it has developed into a prominent organization advancing high-quality, effective and efficient cancer care for patients throughout the world. As I have served as Chair of the NCCN Guidelines for Non-Small Cell Lung Cancer for 20 years, the first guideline, in 1996, had 40 references; currently, version 5.2015 has 718. Wow! Twenty-years ago the guidelines weren't on the 'radar screen' of any physician's treating patients with cancer; now there isn't a place in the world where I have visited, talking about lung cancer, that physicians do not mention or quote the guidelines. Fantastic! Continue the great work!"

\section{Robert W. Carlson, MD (2010)}

"When we developed the agenda for the very first NCCN Breast Cancer Guidelines Panel meeting in 1995, we thought that there would be multiple differences of opinion among the NCCN institutions about how to treat breast cancer. We began the agenda with those clinical situations where we thought there would be most agreement, and then moved into those where we thought there would be controversy and disagreement. At the end of the day, we realized that there was actually unanimous agreement on almost all of the guideline recommendations! In retrospect, we shouldn't have been surprised. When disease experts look at the scientific evidence objectively and inclusively, there should be and is generally agreement.

The early NCCN Guidelines program's principles and processes established under the leadership of Rodger Winn were visionary and laid the foundation for clinically useful, dynamic, and credible NCCN Clinical Practice Guidelines that now
JNCCN Staff

Rodger Winn Award Recipients:

2009

David S. Ettinger, MD

The Sidney Kimmel Comprehensive Cancer Center at Johns Hopkins

2010 Robert W. Carlson, MD Stanford Cancer Institute

2011 Al B. Benson III, MD Robert H. Lurie Comprehensive Cancer Center of Northwestern University

2012 Andrew D. Zelenetz, $M D, P h D$ Memorial Sloan Kettering Cancer Center

2013 Robert J. Morgan, MD City of Hope Comprehensive Cancer Center

2014 Paul F. Engstrom, MD Fox Chase Cancer Center

2015

Daniel G. Coit, MD Memorial Sloan Kettering Cancer Center

The ideas and viewpoints expressed in this commentary are those of the author and do not necessarily represent any policy, position, or program of NCCN. 
define best practices in the United States and many other parts of the world. The concepts of evidence-based recommendations, whenever possible; development by multidisciplinary teams of experts; and assurance of patient and physician choice have been central to the NCCN Guidelines process over the years. Being a part of this process has been an honor and a privilege!"

\section{Al B. Benson III, MD (2011)}

"Rodger Winn's contributions to NCCN and to the concept of guidelines development, administrative infrastructure, and methodology in general are immeasurable. His vision clearly helped to fill a void by cultivating an essential tool to assist clinicians' efforts to provide timely, state of the art, evidence-based care for patients with cancer. The guideline derivative products, including the Drugs \& Biologics Compendium, also have been influential in advancing the most effective treatment options for patients. As someone who was a recipient of Rodger's mentorship as the first NCCN Guidelines Panels were organized, it has been a great honor to be designated an NCCN Winn Awardee."

\section{Robert J. Morgan, MD (2013)}

"The National Comprehensive Cancer Network has grown to be an internationally recognized organization whose influence has continued to improve the medical care of patients with cancer. At the beginning of the NCCN Guidelines program, I don't think that anyone foresaw the great impact that these efforts would have on cancer care. It is largely through the foresight of Rodger Winn, MD, and Bill McGivney, $\mathrm{PhD}$, that the guidelines have attained the prominence and influence that make them a major source for referencing the best in care. The entire NCCN staff works very hard to maintain the excellence of the guidelines process. It has been and continues to be one of the highlights of my career to have had the privilege to work with such a distinguished team of physicians and NCCN staff colleagues. I foresee that the continued efforts to improve the guidelines and the process will result in increased international recognition, importance, and improvements in the lives of our patients."

\section{Paul F. Engstrom, MD (2014)}

"As a member of Rodger Winn's inaugural Guidelines Steering Committee and as Chair of the original NCCN Guidelines Panel for Colon, Rectal, and Anal Cancers writing committee, I have witnessed the growing influence and acceptance of NCCN Guidelines. Developing global guidelines was a challenges because a multidisciplinary team consisting of faculty from the original 13 NCCN Member Institutions had to actually agree on complex treatment plans for patients referred to our respective institutions. As the development process has become more sophisticated, the guidelines have become the standard of treating cancer throughout the United States and in many foreign countries. In addition, the guidelines have also become the basis for reimbursement by CMS for chemotherapy of advanced cancer.

I take great personal pride in the fact that the NCCN Guidelines provide an ever increasing opportunity for NCCN to improve patient care and influence physician behavior. Simply stated, the time and effort that I devoted to these NCCN-related projects is one of the most satisfying and rewarding accomplishments in my career in oncology." 
The Rodger Winn Award

\section{Daniel G. Coit, MD (2015)}

"I can recall quite vividly the initial face-to-face meeting of our NCCN Melanoma Panel, now some 20 years ago. Clinical experts from around the country had enthusiastically agreed to gather and create a basic framework to guide the treatment of patients with melanoma, from the earliest to the most advanced stages. As would be expected with such a gathering of highly opinionated experts, the tenor of that initial meeting was spirited and often quite contentious. Ultimately, we achieved consensus and made progress under the steady helm and perceptive, calm, intelligent leadership of our first Panel Chair, Alan Houghton. Rodger Winn was also present at that and many subsequent meetings, gently guiding the process, but not the content, of the discussions, to ensure the development of a consistent high-quality product across all disease sites. When we were on the verge of getting lost in passionate debates over minutiae, a quiet word or two from Rodger would always return our focus to the big task at hand.

I suspect that few, if any, of us were really aware at the time of how nationally and internationally impactful these guidelines would eventually become, as the initial vision of the NCCN founders has become a reality over the ensuing 2 decades. I think that now, with the perspective of time, many on the NCCN Melanoma Panel have come to realize that our work at NCCN is as important as anything we have ever accomplished outside of the clinical practice of medicine. Certainly for me, personally, I will always consider being named as the 2015 recipient of the Rodger Winn award, on the 20th anniversary of that first meeting, as one of the single greatest honors of my career." 